\title{
Three-Dimensional Low Symmetry Mesoporous Silica Structures Templated from Tetra-Head Group Rigid Bolaform Quaternary Ammonium Surfactant
}

Shaodian Shen ${ }^{1,2}$, Alfonso E. Garcia-Bennett ${ }^{3}$, Zheng Liu, Qingyi Lu ${ }^{1}$, Yifeng $\mathrm{Shi}^{1}$, Yan Yan ${ }^{1}$, Chengzhong $\mathrm{Yu}^{1}$, Weichang $\mathrm{Liu}^{2}, \mathrm{Ya} \mathrm{Cai}^{2}$, Osamu Terasaki ${ }^{3}$, Dongyuan $\mathrm{Zhao}^{1}$ *

\section{Supplementary Information.}

Table S1. Observed and calculated X-ray d-spacing of the tetragonal P4/mmm symmetry for the FDU-11 silica material $(a=8.512 \mathrm{~nm}, c=5.262 \mathrm{~nm})$.

\begin{tabular}{|l|l|r|}
\hline$h k l$ & $d$ (observed) $(\mathrm{nm})$ & $d$ (calculated) $(\mathrm{nm})$ \\
\hline 110 & 5.889 & 6.019 \\
\hline 001 & 5.262 & 5.262 \\
\hline 210 & 3.808 & 3.807 \\
\hline 220 & 2.935 & 3.009 \\
\hline 002 & 2.695 & 2.631 \\
\hline 320 & 2.380 & 2.361 \\
\hline 400 & 2.028 & 2.128 \\
\hline 302 & 1.954 & 1.929 \\
\hline
\end{tabular}

Table S2. Observed and calculated SAXS $d$-spacings of the tetragonal $P 4 / \mathrm{mmm}$ symmetry for the FDU-11 silica material $(a=8.462 \mathrm{~nm}, c=5.224 \mathrm{~nm})$.

\begin{tabular}{|c|r|r|}
\hline \multicolumn{1}{|l|}{$h k l$} & $d$ (observed) $(\mathrm{nm})$ & $d$ (calculated) $(\mathrm{nm})$ \\
\hline 100 & 8.622 & 8.462 \\
\hline 110 & 5.999 & 5.983 \\
\hline 001 & 5.224 & 5.224 \\
\hline 210 & 3.784 & 3.784 \\
\hline 201 & 3.241 & 3.288 \\
\hline 220 & 2.951 & 2.992 \\
\hline 002 & 2.607 & 2.611 \\
\hline 112 & 2.390 & 2.394 \\
\hline 400 & 2.080 & 2.115 \\
\hline 410 & 2.043 & 2.052 \\
\hline 302 & 1.938 & 1.916 \\
\hline 312 & 1.869 & 1.869 \\
\hline
\end{tabular}


Table S3. Observed and calculated X-ray $d$-spacings of the orthorhombic Pmmm symmetry for the FDU-13 silica material $(a=9.80 \mathrm{~nm}, b=5.889 \mathrm{~nm}, c=3.743 \mathrm{~nm})$.

\begin{tabular}{|l|r|r|}
\hline$h k l$ & $d$ (observed) $(\mathrm{nm})$ & $d$ (calculated) $(\mathrm{nm})$ \\
\hline 010 & 5.889 & 5.851 \\
\hline 110 & 5.048 & 5.024 \\
\hline 001 & 3.743 & 3.743 \\
\hline 101 & 3.451 & 3.497 \\
\hline 011 & 3.078 & 3.153 \\
\hline 120 & 2.832 & 2.803 \\
\hline 220 & 2.517 & 2.512 \\
\hline 121 & 2.248 & 2.244 \\
\hline 002 & 1.872 & 1.872 \\
\hline
\end{tabular}

Table S4. Observed and calculated SAXS $d$-spacings of the orthorhombic Pmmm symmetry for the FDU-13 silica material $(a=9.806 \mathrm{~nm}, b=5.671 \mathrm{~nm}, c=3.662 \mathrm{~nm})$.

\begin{tabular}{|l|r|r|}
\hline$h k l$ & $d$ (observed) $(\mathrm{nm})$ & $d$ (calculated) $(\mathrm{nm})$ \\
\hline 010 & 5.671 & 5.671 \\
\hline 110 & 4.911 & 4.909 \\
\hline 001 & 3.662 & 3.662 \\
\hline 101 & 3.351 & 3.431 \\
\hline 011 & 3.049 & 3.078 \\
\hline 020 & 2.828 & 2.835 \\
\hline 120 & 2.717 & 2.724 \\
\hline 211 & 2.652 & 2.606 \\
\hline 220 & 2.527 & 2.545 \\
\hline 301 & 2.454 & 2.439 \\
\hline 021 & 2.266 & 2.242 \\
\hline 121 & 2.163 & 2.186 \\
\hline
\end{tabular}

